\title{
Comparison of envelope detection techniques in Coherence Scanning Interferometry
}

\author{
G.Gianto, ${ }^{*}$ F. Salzenstein, P. Montgomery
}

ICube Laboratory, University of Strasbourg-CNRS, 23 rue du Loess, 67037 Strasbourg, France

*Corresponding author: gqianto@unistra.fr

Received XX Month XXXX; revised XX Month, XXXX; accepted XX Month XXXX; posted XX Month XXXX (Doc. ID XXXXX); published XX Month XXXX

\begin{abstract}
The aim of this work is to make a comparison of the most current signal processing techniques used to analyze the fringe signal in Coherence Scanning Interferometry (CSI), a major technique for optical surface roughness measurement. We focus here on classical AM-FM signal processing algorithms such as Hilbert Transform (HT), Five Sample Adaptive (FSA) and Continuous Wavelet Transform (CWT). We have recently also introduced a new family of compact and robust algorithms using the Teager Kaiser Energy Operator (TKEO). We propose an improved version of TKEO using the combination of different techniques of pre-filtering and demodulation processing to remove the noise and offset component, and to retrieve the fringe envelope to determine the surface height information. In particular, as a pre-filtering approach, we have focused on empirical mode decomposition (EMD) in combination with the Savitzky-Golay filter. An added Gaussian post-filtering is helpful for a precise peak extraction. The experimental results show that TKEO performs better than CWT in terms of computational time and provides a better surface extraction than the HT and FSA. Results have been obtained on synthetic and real data taken from a layer of resin on a silicon (substrate). (C) 2016 Optical Society of America
\end{abstract}

OCIS codes: (100.0100) Image Processing; (100.2650) Fringe analysis; (100.3175) Interferometric Imaging (100.5070) Phase retrieval

\section{INTRODUCTION}

Coherence Scanning Interferometry (CSI) is a major non-contact optical surface profiling technique that makes use of white light interference fringes as an optical probe for measuring surface roughness [1]. CSI is widely used in many fields of materials characterization [2] and surface metrology [3] and has now been extended to medical imaging not only for the surface measurement of skin and tissue but also for internal tomographic imaging using full-field optical coherence tomography (FF-OCT) [4,5]. The basic CSI technique has the advantages of being fast, robust, high resolution and non-destructive. Another main advantage concerns its capability to work without the problem of phase ambiguity in the measurement of step heights greater than $\lambda / 2$, which is unavoidable in monochromatic interferometry measurements $[1,6]$.

In this work we are particularly interested in developing CSI for the high resolution characterization of medium thickness ( $1 \mu \mathrm{m}$ to $20 \mu \mathrm{m})$ transparent, semi-transparent and diffusing layers [2]. For example, we have previously studied inhomogeneous layers of hydroxyapatite and colloids to measure local buried internal structures, layer thickness, and effective refractive index [7]. Such layers are known to be a challenge to measure optically [6] because of the diffuse nature of the materials.

The algorithms commonly used for surface roughness measurement involve processing the fringe signals to identify a single envelope corresponding to the position of the surface at each pixel. These consist of demodulation [8], the HT [9,10], FSA [11] and CWT [12,13]. While CWT algorithms are known to be precise and robust, they are nonetheless time consuming. For the processing of multiple fringe envelopes in FF-OCT, slightly modified versions of the above are used [14]. The challenges here are how to deal with the noise and the offset component and improve the envelope detection extraction performance so that the algorithm provides more precise surface measurement while being quicker in terms of computational time.

Recently, we introduced a new family of compact and robust algorithms using the TKEO [15]. These have the advantages of efficiency and simplicity for tracking the instantaneous amplitude and frequency of AM-FM signals. In the present work we propose an improved version of TKEO using the combination of different techniques of pre-filtering and demodulation processing to remove the noise and offset component, and to retrieve the fringe envelope to determine the surface height and internal structural information. In particular, we use empirical mode decomposition (EMD) [16] in combination with the Savitzky-Golay filter for pre-filtering together with Gaussian postfiltering for peak extraction. The improved performance of the new algorithm is demonstrated on synthetic and real data taken from a layer of resin on a silicon substrate and compared with the results from the classical techniques mentioned.

\section{PRINCIPLES OF FRINGE PROCESSING IN CSI}


The principle of CSI (Fig. 1) is generally based on the scanning of white light interference fringes over the whole depth of the sample along the optical axis. By means of a single vertical scan of the sample, a stack of $\mathrm{XYZ}$ images is generated. In surface roughness measurement, a signal processing technique aims at providing the peak position of a single fringe envelope along $Z$ at each pixel $\left(x_{0}, y_{0}\right)$. This peak position $z_{0}$ corresponds to the height of the surface at each point in the image XY [7]. For tomographic imaging it is necessary to extract several fringe envelopes along $Z$ corresponding to the surface and to buried structures and layers.

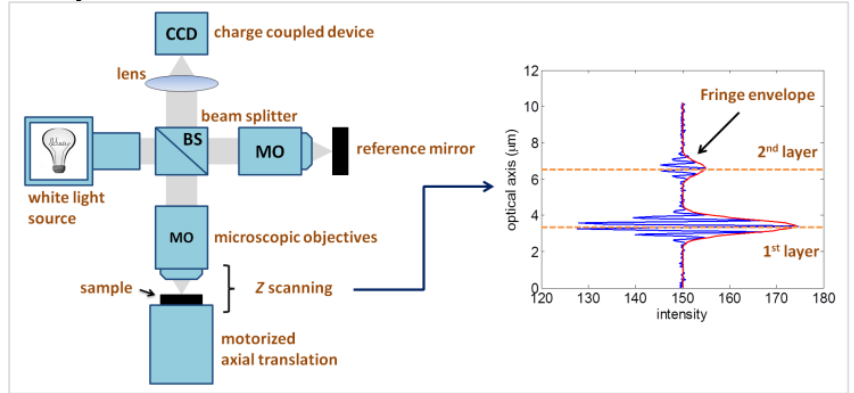

Fig. 1. Typical layout of Coherence Scanning Interferometry using a Linnik objective.

The precise extraction of the fringe envelopes is an essential procedure in the analysis of the white light fringes for correct surface roughness or layer characterization. However, the performance of a given algorithm depends on its sensitivity to the different sources of signal noise and artifacts. Another problem lies in an additive offset component (background) which can appear in the fringe signals during the acquisition process, particularly over large scanning depths. In order to remove this offset component and the additive noise, it is important to filter out both of them before applying fringe envelope detection. Thus, as a pre-processing step, we have evaluated the performance of processing techniques which are commonly used for removing the offset component. As an alternative, we propose the well-known decomposition technique, i.e. the Empirical Mode Decomposition (EMD) technique for tracking the offset component of fringe signals. Contrarily to the wavelet decomposition, this technique does not need any prior assumption regarding the signal properties [16]. Then for noisy signals, we have evaluated two different denoising filters, i.e. the wiener filter and Savitzky-Golay filter to reduce the noise.

The main processing step i.e. envelope detection, is the subject of several signal processing techniques in the literature. In this study, we propose the comparison of the Five Sample Adaptive (FSA) [11], the Hilbert Transform (HT, based on Fast Fourier Transform) $[9,10]$, the Continuous Wavelet Transform [12,13] (CWT), and TKEO [15]. Moreover, we show how the Savitzky-Golay filter improves the performance of TKEO and makes this algorithm more robust to noise. Then we demonstrate how the use of Gaussian curve fitting on the fringe envelope peaks provides a more precise measurement of position.

\section{ANALYSIS OF WHITE LIGHT INTERFERENCE FRINGES}

\section{A. Signal model}

A typical fringe signal obtained from a detector (digital camera) as the OPD is varied through focus in a white light interferometer, has the following form $[10,11]$ :

$$
\begin{aligned}
s(x, y, z)= & a(x, y, z)+b(x, y) g\left(z-z_{0}\right) \\
& . \cos \left(\frac{4 \pi}{\lambda_{0}}\left(z-z_{0}\right)-\alpha(x, y)\right)
\end{aligned}
$$

where the function $s$ corresponds to the intensity signal corresponding to a given point on the sample surface $(x, y)$, where $z$ represents a vertical scanning position along the optical axis in relation to the surface. The factor $a(x, y, z)$ is a non-constant offset intensity related to the reference and object beam intensities, $b(x, y)$ is the fringe contrast, $g(z)$ is the fringe envelope function related to the spectral profile of the white light source, and $\lambda_{0}$ is the mean wavelength of the light source. The phase offset related to the phase change on reflection is represented by $\alpha(x, y)$.

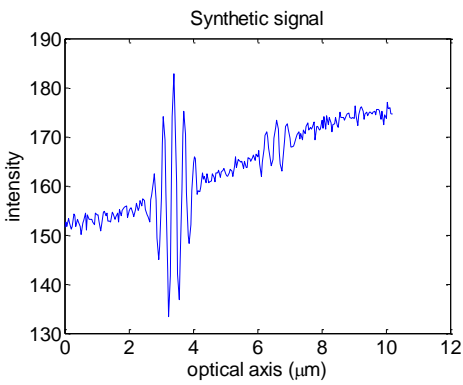

Fig. 2. Structure of white light interference fringe.

\section{B. White light interference fringe analysis}

In order to obtain the surface height information, in the techniques developed in this work, we have performed-three main steps consisting of pre-filtering, envelope detection and post-filtering (Fig. 3).



Fig. 3. The procedure of white light interference fringe analysis.

\section{Pre-filtering}

The pre-filtering is essential in order to filter out the offset component and the noise. In coherence scanning interferometry, the first order derivative based on the central (finite) difference is a common method used to remove the offset (low frequency variation of the intensity). By removing the values of the signal where their adjacent/neighbourhood values are almost equal, the first derivative effectively removes the lower frequencies of the signal, i.e. the offset component. However, it can fail in the presence of significant noise. For this reason, we have tested more robust filters such as the Savitzky-Golay or wiener family in combination with the derivative.

Alternatively, we have performed the well-known decomposition technique known as Empirical Mode Decomposition (EMD). The EMD method has the ability to decompose a signal into oscillatory components, called intrinsic mode functions (IMFs). The principle of EMD is the use of a repeated shifting process on the analyzed signal. For a given signal $x(\mathrm{t})$, all extrema (local maxima and minima) are detected and connected by an interpolating technique, such as the cubic spline, that produces respectively the upper envelope $e_{\max }(t)$ and the lower envelope $e_{\min }(t)$. The average of the two envelopes is then computed, $m(t)=\left(e_{\max }(t)+e_{\min }(t)\right) / 2$ and the remaining detail $d(t)=x(t)-m(t)$, is extracted. The above procedure is repeated in order to obtain the first intrinsic mode functions (IMFs), $d_{1}(t)$ which satisfies two conditions: 
a) The number of extrema (the sum of the maxima and minima) and the number of zero-crossings must either be equal or differ at most by one.

b) At any point of an IMF the mean value of the envelopes of the local maxima and minima is zero.

After the $1^{\text {st }}$ IMF is obtained, a residue $r(t)=x(t)-d_{1}(t)$ is computed and processed as a new signal $x(t)$. The same procedure is applied to the new signal until other IMFs, $d_{2}(t), d_{3}(t), d_{\mathrm{N}}(t)$ are obtained. Finally, the result of decomposition using the EMD method from the $x(t)$ signal yields $N$ component of IMF's and a residue.

$$
x(t)=\sum_{k=1}^{N} d_{k}(t)+r(t)
$$

The lower-order IMF's typically represents fast oscillation modes, while the higher order IMF's and the residue typically represent slow oscillation modes. Due to these characteristics, the EMD technique seems to be well adapted to suppress the slowly varying offset.

\section{Envelope Peak Detection}

In the CSI technique, the precision of the measurement of surface roughness and microscopic surface shape depends on the ability to detect the peak of the fringe envelope. The technique involves scanning the location of zero optical path difference (OPD), which indicates the surface height position. For this purpose, an envelope of the signal intensity is extracted, and its local maximum location corresponds to a zero OPD i.e., the surface height. The current detection methods used are the HT technique, FSA , CWT and TKEO [9-13,15]. We recall these methods in section $\mathrm{C}$ below.

\section{Post-filtering}

For noisy fringe signals, even though pre-filtering is used to suppress the noise, the fringe structure can still appear in the resulting fringe envelope. Because of this, a smoothing filter such as a cubic spline is used to improve the envelope peak detection. Then, the curve fitting method using Gaussian fitting is implemented in order to determine more precisely the envelope peak.

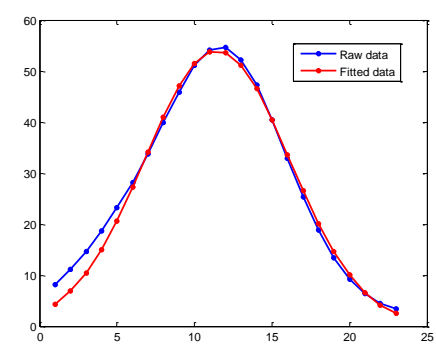

Fig. 4. The curve fitting method using a Gaussian estimation with 22 points near to the peak.

\section{Fringe envelope detection techniques}

In the following the different techniques used to retrieve the fringe envelope in this work are presented.

\section{Hilbert Transform (HT)}

For fringe envelope extraction, the Hilbert Transform (HT) works by suppressing the high frequency component of the interference fringe signal in order to obtain the low frequency part. The main steps of the Hilbert Transform technique using a Fast Fourier Transform (FFT) calculation are outlined in Fig. 5. The FFT is applied to the signal, as shown in Fig. 5(a). The spectrum of the signal obtained by the FFT is illustrated in Fig. 5(b). The FFT coefficients that correspond to negative frequencies are replaced with zeros as shown in Fig. 5(c). Finally, the fringe envelope as shown in Fig. 5(d) is extracted by calculating the inverse FFT of the positive frequency packet in Fig. 5(c).

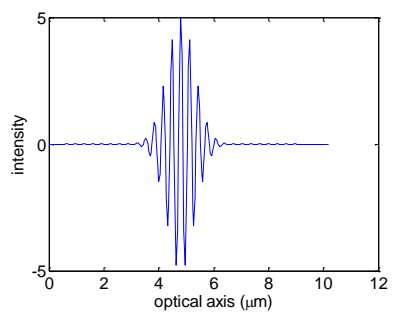

(a)

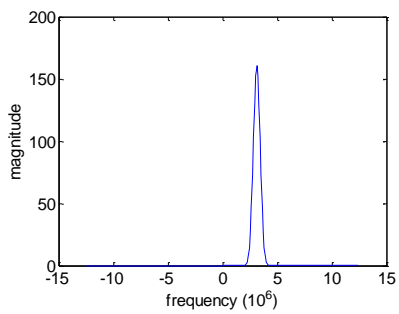

(c)

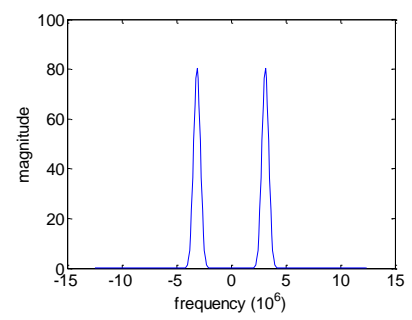

(b)

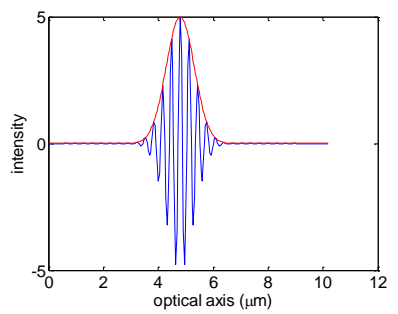

(d)
Fig. 5. Fringe envelope detection process using Hilbert Transform technique: (a) fringe signal intensity generated by CSI; (b) spectrum of Fourier transform calculated by FFT; (c) filtering out negative frequencies; (d) extracted fringe envelope by inverse Fourier transform.

\section{Five Sample Adaptive (FSA)}

The Five Sample Adaptive (FSA) algorithm proposed by Larkin [11] is a fast and simple method which has been commonly applied in CSI in order to determine the envelope of a fringe signal. At each sampling position, the value of the envelope is computed using four neighbouring sampling positions. The calculated fringe envelope is performed by:

$$
4 A^{2} \sin ^{4} \phi=\left(I_{2}-I_{4}\right)^{2}-\left(I_{1}-I_{3}\right)\left(I_{3}-I_{5}\right)
$$

where $A$ is the amplitude value at a given sample, $\phi$ is the phase shift due to the scanning step, $I_{3}$ is the local position, while $I_{1}, I_{2}, I_{4}, I_{5}$ are the neighbouring sampling positions.

For values of $\phi$ close to $90^{\circ}$, the equation [4] can be written as:

$$
A^{2}=\frac{1}{4}\left[\left(I_{2}-I_{4}\right)^{2}-\left(I_{1}-I_{3}\right)\left(I_{3}-I_{5}\right)\right]
$$

Due to its simplicity, the FSA technique is competitive in terms of computation time. However, this algorithm requires the values of $\phi$ to be close to $90^{\circ}$, for accurate measurement.

\section{Continuous Wavelet Transform (CWT)}

The Wavelet transform is a time-frequency analysis technique which has been widely used in signal analysis and processing $[17,18,19]$. Due to its ability to decompose locally the signal into different scale/frequencies, CWT highlights the region of interest, where the local frequency corresponds to the carrier frequency of the CSI signal. Moreover, the CWT method seems to be robust to noise $[17,20]$, providing accurate measurements of the surface.

The CWT transform function of a fringe signal $s(z)$ can be expressed as:

$$
W(a, b)=\frac{1}{|a|^{1 / 2}} \int_{-\infty}^{\infty} s(z) \psi\left(\frac{z-b}{a}\right) d z
$$

where $W(a, b)$ represents the CWT coefficient function, $\psi(z)$ is a continuous function in time and frequency domain called the mother wavelet, $a$ is the scale factor, and $b$ is the shift factor. The well-known complex Morlet wavelet $[6,13,21,22]$ has been chosen as the mother wavelet since its properties have strong similarities to the fringe signal, since the wavelet function corresponds to a Gaussian envelope modulated by a sinusoidal function. 


$$
\psi(z)=e^{i \omega z} e^{\left(-z^{2} / 2\right)}
$$

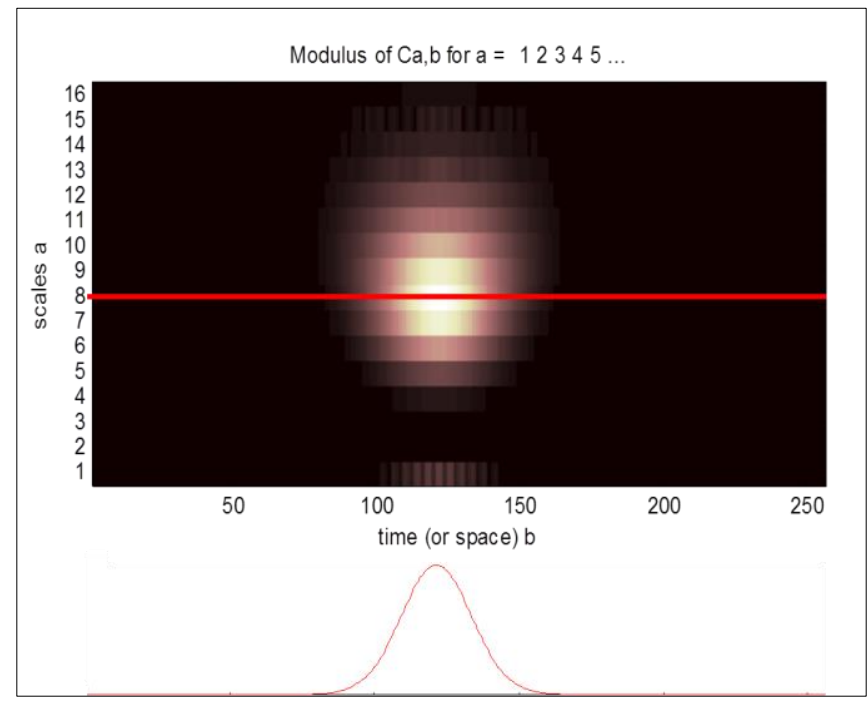

Fig. 6. The modulus of coefficient CWT of the fringe signal.

In order to compute each coefficient $W(a, b)$, the fringe signal is convolved with a set of complex Morlet wavelets. As a function of scale factors $a$ and shift $b, W(a, b)$ can be represented by a scalogram, as shown in Fig. 6. The fringe envelope corresponds to the absolute modulus of the maximum correlation coefficient as shown by the red line in the scalogram.

\section{Teager Kaiser Energy Operator (TKEO)}

The Teager Kaiser Energy Operator (TKEO) [23-25] is an operator that tracks the instantaneous energy of a signal. This non linear energy operator and its 1D/2D discrete versions has found applications in various fields of signal and image processing [25,26,27] due to its success in analysing and demodulating AM-FM signals with high resolution, simplicity, and efficiency. In its discrete version, only three samples are required at each time instant. In the continuous case, the Teager Kaiser Energy Operator is defined by:

$$
\begin{aligned}
\psi[s(z)] & =\&(z)-s(z) z z) \\
& =\left(\frac{\partial s(z)}{\partial z}\right)^{2}-s(z)\left(\frac{\partial^{2} s(z)}{\partial z^{2}}\right)
\end{aligned}
$$

where $s(z)$ is the signal, $\&(z)$ and $(z)$ means the first derivative and the second derivative of $s$ respectively. A discrete forward and backward approximation of the derivatives of equation (7) leads to the discrete TKEO:

$$
\psi[s(n)]=s^{2}[n]-s[n-1] s[n+1]
$$

Let us consider a mono-component continuous time AM-FM signal:

$$
s(t)=a(t) \cos (\phi(t))
$$

where $a(t)$ represents the spatially varying amplitude and $\phi(t)$ is the phase signal. The output of the Teager Kaiser Energy Operator applied to $s(t)$, is given by $\psi[s(t)] \approx[a(t) \not(t)]^{2}$, where $\not(t)$ is the instantaneous phase. Moreover, the Energy Separation Algorithm (ESA), estimates the instantaneous envelope $a(t)$ and the instantaneous phase $\not(t)$ as follows:

$$
|a(t)| \approx \frac{\psi[s(t)]}{\sqrt{\psi[\&(t)]}} ; \quad|\not t(t)| \approx \sqrt{\frac{\psi[\&(t)]}{\psi[s(t)]}}
$$

The main disadvantage of the Teager Kaiser Energy Operator lies in its sensitivity to noise, due to the successive derivations. In the context of noisy data, a filter such as the Savitzky-Golay approach, improves the robustness of TKEO.

\section{PERFORMANCE OF THE DIFFERENT METHODS}

Some simulations have been performed in order to compare the different techniques for surface roughness detection in CSI. The general experimental procedure is outlined in Fig. 7:

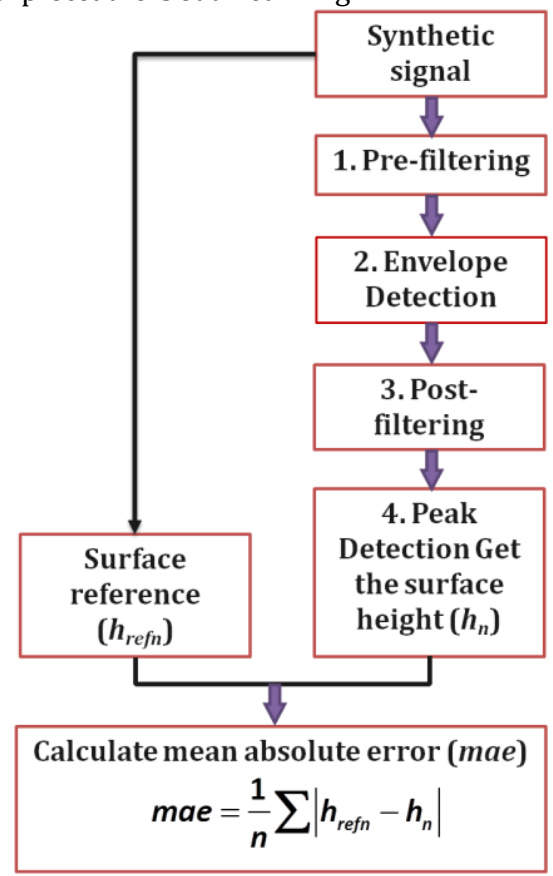

Fig. 7. The procedure of evaluation of algorithm performance.

The synthetic signal we have used in this simulation to test all the cited algorithms is based locally on the general model expressed by the equation (1) along the optical axis Z, with a $40 \mathrm{~nm}$ sampling step, for each lateral position X. The resulting XZ image is shown in Fig. 8. The synthetic image represents a transparent layer on a substrate i.e, two surfaces. An additive non linear offset and the Gaussian noise are added to this synthetic data since they commonly appear in the fringe signal during the acquisition process and lead to a recution in the precision of surface measurement.

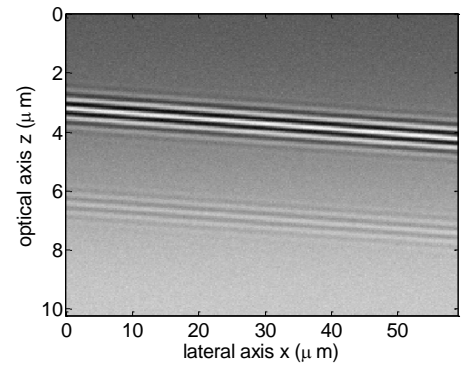

Fig. 8. Synthetic signal (XZ image) 256 x 256 pixel.

The performance of each approach is then evaluated by the following statistical parameter of mean absolute error (mae): 


$$
\text { mae }=\frac{1}{n} \sum\left|h_{r e f n}-h_{n}\right|
$$

where $h_{\text {refn }}$ represents the reference surface level, and $h_{\mathrm{n}}$, is its estimated height along the lateral axis-X.

In addition, these algorithms have also been evaluated on a second synthetic fringe signal, containing two transparent curved surfaces (Fig. 9 (a)) which can be encountered in some cases. Finally, we have tested them on a real fringe signal (see Fig. 9 (b)), which corresponds to a resin layer on Silicon. This real image is chosen due to the presence of a non linear offset and the noise on the fringes so that the advanced signal processing is required to provide correct surface extraction.

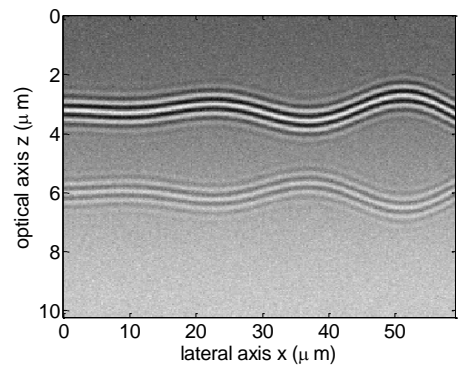

(a)

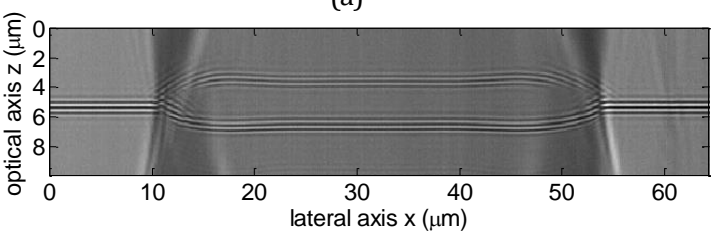

(b)

Fig. 9. (a) Synthetic fringe signal of transparent curve surface and (b) real fringe signal of resin on $\mathrm{Si}$.

For a flat surface, such as the resin on $\mathrm{Si}$, we propose to evaluate the performance using the total (or peak-valley) roughness $R_{t}$ and arithmetic roughness $R_{a}$ of the profile. The maximum height of the profile $R t$, is computed as follows [28]:

$$
R_{t}=\left|z_{\max }-z_{\min }\right|
$$

Where $Z_{\max }$ (resp. $Z_{\min }$ ) represents the highest (resp. the lowest) peak of the surface profile. $R_{a}$ is the average deviation of the roughness profile height $z_{j}$ from a mean line $\bar{z}$ over the evaluation length $N$ [29].

$$
R_{a}=\frac{1}{N} \sum_{j=1}^{N}\left|z_{j}-\bar{z}\right|
$$

\section{EXPERIMENTAL RESULTS}

\section{A. Analysis of signal processing procedure}

\section{Pre-filtering step}

In order to remove the offset and reduce the noise, we have compared in our study three different algorithms which combine the derivative/EMD technique and denoising filter (Savitzky-Golay/Wiener $[30,31,32])$. The corresponding algorithms are referred to with the following names: I) Pre-filter 1: combination of derivative and Wiener filter ii) Pre-filter 2: combination of derivative and Savitzky-Golay filter and iii) Pre-filter 3: combination of EMD and Savitzky-Golay filter. Regarding the removal of the offset, two procedures are considered: a) the classical centered derivative, which has been chosen for its stability [33], and b) as an alternative method, the EMD which helps to remove the low frequency component given by the higher order IMF's and the residue [16]. On the other hand, to process the noisy data, the SavitzkyGolay filter is compared with the classic Wiener filter [31], using a local window around each sample. Based on empirical data, the window length of 5 seems to optimize the performance for this approach (with a
$40 \mathrm{~nm}$ sampling step). Concerning the Savitzky-Golay filter, we have applied it along the lateral axis using a third order polynomial. Based, on the data used, this parameter seems to ensure the better robustness to noise.

Figure 10 shows a comparison of the results of the performance of pre-filter 1 , pre-filter 2 , and pre-filter 3.

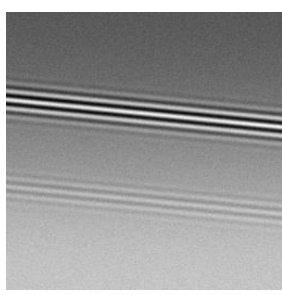

(a)

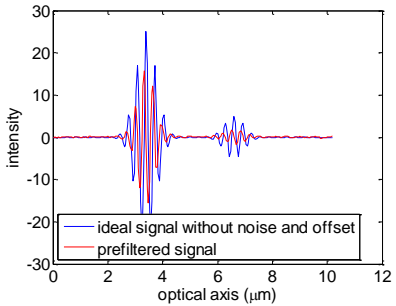

(c)

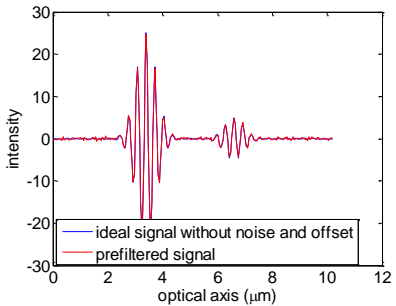

(e)

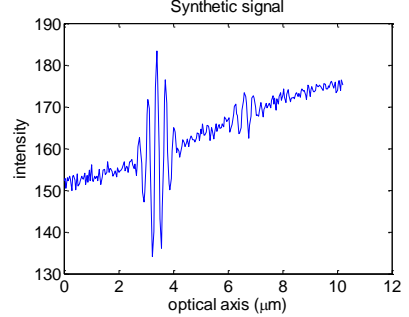

(b)

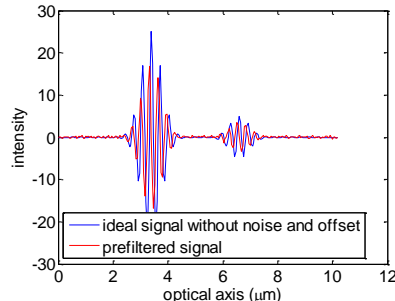

(d)
Fig. 10. Synthetic signal with noise $\sigma=20 \%$ in the form of (a) XZ image (256 x 256 pixels) and (b) 1D (the intensity value along the optical axis z); prefiltered signal resulting from: (c) pre-filter 1; (d) pre-filter 2; (e) pre-filter 3.

It can be observed in Fig. 10, that the three algorithms provide competitive results for the offset removal and noise reduction However, they have different capabilities for maintaining the fringe signal intensities. Comparisons are made between the fringe signal intensities of the pre-filtered (red line, Fig. 10) and the ideal signals i.e. without the offset and noise (blue line, Fig. 10). As illustrated in Fig. 10 (c), the resulting amplitude of the fringe signal using pre-filter 1 , is clearly lower than the ideal synthetic fringe signal (without the offset or the noise). This means that a degradation of the intensities occurs during the pre-filtering process. Similar results are also obtained using pre-filter 2 (Fig. 10(d)), although it maintains better the fringe signal intensities of the second layer. The decrease in the intensity may seriously degrade the performance of the algorithm in order to extract the fringe envelope, particularly in the area where the SNR is low. As illustrated in Fig. 10(e), the pre-filter 3 is able to remove the offset and reduce the noise, while almost maintaining the amplitude of the fringe signal.

\section{Envelope Detection}

The next step concerns the local envelope extraction of the pre-filtered signal, also known as fringe envelope detection. This step may be considered as an important part in white light fringe analysis because the information is contained in the peak location of the envelope. We now propose using the previously mentioned algorithms (HT, FSA, CWT and TKEO) $[9-13,15]$ to identify the fringe envelope of such data and to estimate the relative effectiveness of each one. 
The results in Fig. 11 illustrate the fringe envelope detection according to the different techniques for a multilayer fringe signal, in the presence of noise.

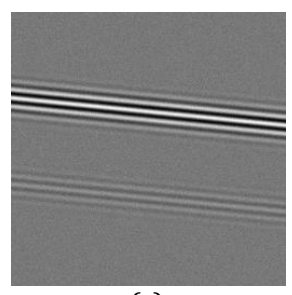

(a)

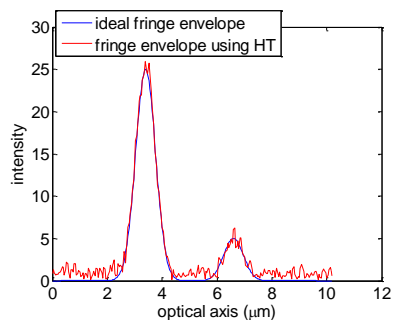

(c)

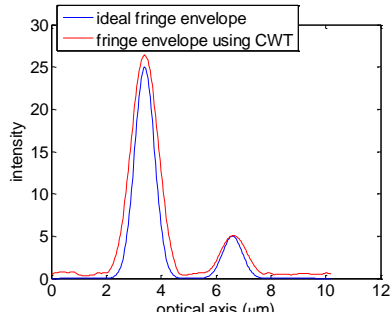

(e)

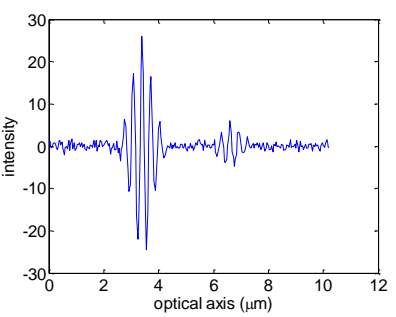

(b)

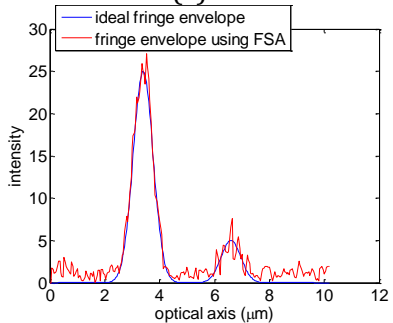

(d)

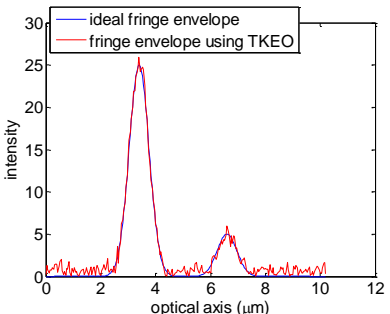

(f)
Fig. 11. The multilayer fringe signal with noise of $\sigma=20 \%$ in the form of (a) XZ image (256 x 256 pixel) and (b) 1D (the intensity value along the optical axis $\mathrm{z}$ ), is processed using different envelope detection: (c) HT; (d) FSA; (e) CWT; (f) TKEO.

As shown in Fig. 11, it can be observed that the CWT algorithm provides the best results, in extracting the fringe envelopes and their associated carrier frequencies for the detection of both surfaces. Moreover, the technique is very robust to noise, giving a signal to noise ratio (SNR) over 25 measurements of $54.3 \mathrm{~dB}$ for the $1^{\text {st }}$ envelope and $47.2 \mathrm{~dB}$ for the $2^{\text {nd }}$ envelope (Table 1). The other techniques (HT, FSA, TKEO) reduce to a less extent the artifacts on the fringe envelope (Fig. 11(c-d-f) and Table 1). Higher values of the signal to noise ratio represent the presence of less artifacts.

Table 1. The signal to noise ratio of 25 measurements for the different techniques

\begin{tabular}{ccccccccc}
\hline & \multicolumn{2}{c}{ HT } & \multicolumn{2}{c}{ CWT } & \multicolumn{2}{c}{ FSA } & \multicolumn{2}{c}{ TKEO } \\
\hline Envelope & $1^{\text {st }}$ & $2^{\text {nd }}$ & $1^{\text {st }}$ & $2^{\text {nd }}$ & $1^{\text {st }}$ & $2^{\text {nd }}$ & $1^{\text {st }}$ & $2^{\text {nd }}$ \\
\hline SNR $(d B)$ & 28.1 & 15.2 & 54.3 & 47.2 & 23.7 & 11.8 & 28.9 & 15.6 \\
\hline
\end{tabular}

\section{Post-filtering}

In order to improve the robustness to noise of the new procedures for envelope detection, we have also combined some post-filtering, consisting of cubic spline smoothing [26] and Gaussian curve fitting. The cubic smoothing spline helps to efficiently reduce the noise and also to improve the envelope detection using HT, FSA or TKEO (Fig. 12). However, the buried interface i.e. the second fringe envelope with the lowest level still contains some artifacts (see Fig. 12).

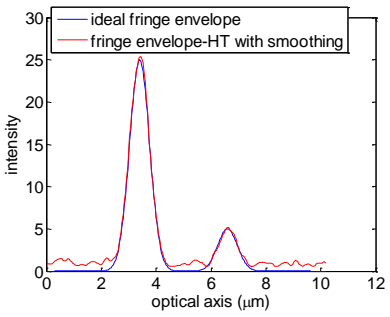

(a)

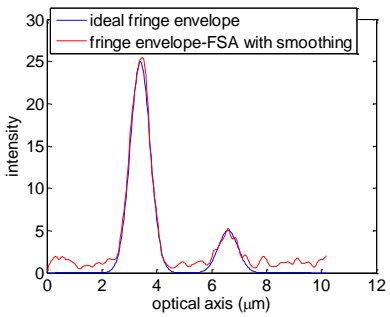

(b)

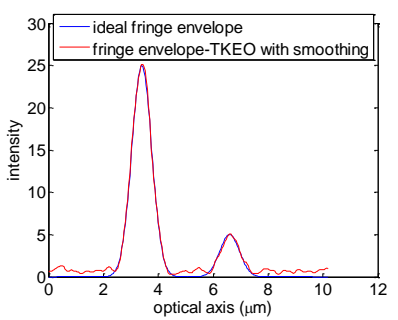

(c)
Fig. 12. The result of smoothing for fringe envelope of: (a) FT; (b) FSA; (c) TKEO.

Finally, an additional Gaussian curve fitting is used to locate the fringe envelope peak position (Fig. 13), and so determine more precisely the height of the surfaces. This additional procedure in particular improves the performance of the buried interface detection.

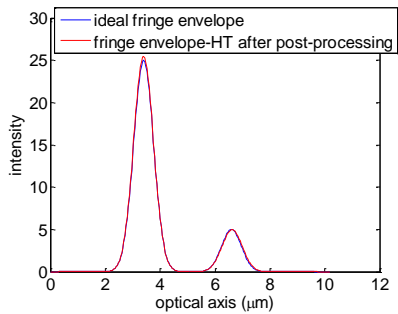

(a)

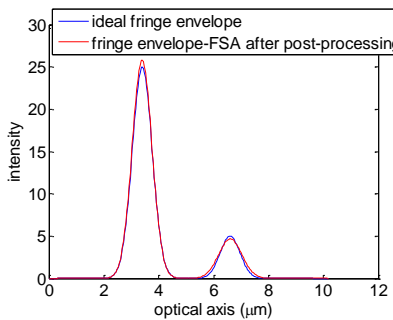

(b)

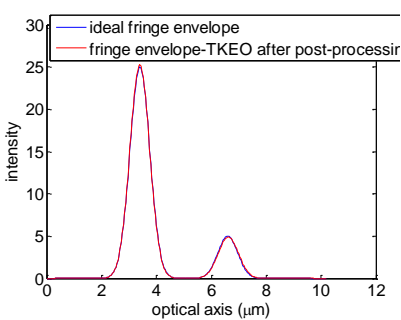

(c)
Fig. 13. The fringe envelope after post-filtering result of smoothing for fringe envelope of: (a) FT; (b) FSA; (c) TKEO.

To improve the axial sensitivity, we used an interpolation method on the Gaussian fitting for the post-processing. The results provide a significant improvement in terms of the precision of surface extraction. as represented by the smaller value of mae in Table 2,3,4 than if only Gaussian fitting was used without interpolation.

\section{Estimation of height of the surface}

From the previous processing steps, we finally derive the height of each surface at a given point of the sample surface. At each lateral site of an $\mathrm{XZ}$ image ( $\mathrm{X}$ being the lateral axis, while $\mathrm{Z}$ being the optical one), we repeat the procedures (pre-filtering, envelope detection and postfiltering) in order to locate the surfaces. The results in Fig. 14 illustrate the final estimated surfaces. The red line represents the first surface, while the green line represents the buried interface. 


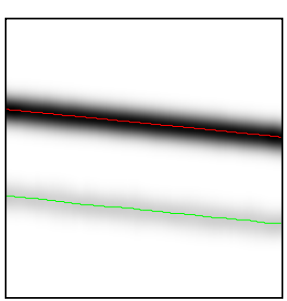

(a)

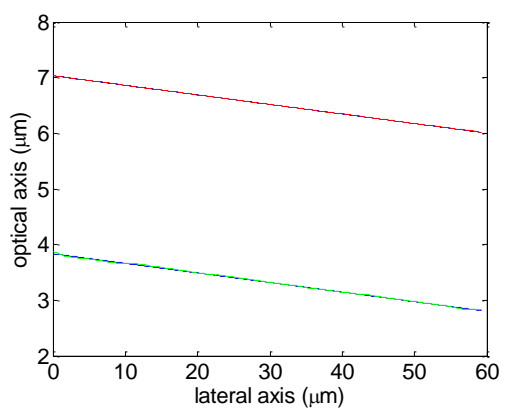

(b)

Fig. 14. (a) The fringe envelope peak positions and (b) their conversion into surface heights.

\section{B. Performance comparison using synthetic images}

For performance the evaluation, the two synthetic fringe images describe previously were used: (1) a flat transparent layer and (2) an undulating transparent layer, as shown in Fig. 8 and 9(a). The algorithms have been implemented using a Matlab program. Tables 1, 2 , and 3 summarize the results as follows: Tables 1, 2 and 3 correspond respectively to the pre-filter 1,2 and 3 , the related algorithms being respectively denoted by A, B, C, D (Table 2), E, F, G, H (Table 3), and I, J, $\mathrm{K}, \mathrm{L}$ (Table 4). For each table, we have indicated the envelope technique associated with each procedure (for instance A, E, and I are related to the Hilbert Transform, while B, F, and J use FSA etc). The same postprocessing mentioned in section 3 , has been applied. For each procedure, the simulations have been calculated with different levels of noise. The performance of each procedure has been quantitatively evaluated by the measurement of the calculation time and the error rate between the original surface and the estimated one (for both surface layers).

Table 2. Experiment result using pre-filter 1 and different envelope detection techniques (nm)

\begin{tabular}{|c|c|c|c|c|c|c|c|c|}
\hline & \multicolumn{2}{|c|}{ A } & \multicolumn{2}{|c|}{$\mathrm{B}$} & \multicolumn{2}{|c|}{$\mathrm{C}$} & \multicolumn{2}{|c|}{$\mathrm{D}$} \\
\hline $\begin{array}{l}\text { Pre- } \\
\text { filt. }\end{array}$ & \multicolumn{8}{|c|}{$\begin{array}{l}\text { Suppress the offset: } 1^{\text {st }} \text { order central derivative } \\
\text { Suppress the noise: Wiener filter }\end{array}$} \\
\hline $\begin{array}{l}\text { Env. } \\
\text { Det. }\end{array}$ & \multicolumn{2}{|c|}{ HT } & \multicolumn{2}{|c|}{ FSA } & \multicolumn{2}{|c|}{ TKEO } & \multicolumn{2}{|c|}{ CWT } \\
\hline $\begin{array}{l}\text { Post } \\
\text {-filt. }\end{array}$ & \multicolumn{8}{|c|}{$\begin{array}{l}\text { Smooth the amplitude envelope using cubic smoothing spline } \\
\text { Correct the peak curve using Gaussian estimation and interpolation }\end{array}$} \\
\hline \multicolumn{9}{|c|}{ mean absolute error (nm) } \\
\hline $\begin{array}{l}\text { nois } \\
e(\sigma)\end{array}$ & $\begin{array}{c}1^{\text {st }} \\
\text { surf. }\end{array}$ & $\begin{array}{c}2^{\text {nd }} \\
\text { surf. }\end{array}$ & $\begin{array}{c}1^{\text {st }} \\
\text { surf. }\end{array}$ & $\begin{array}{c}2^{\text {nd }} \\
\text { surf. }\end{array}$ & $\begin{array}{c}1^{\text {st }} \\
\text { surf. }\end{array}$ & $\begin{array}{c}2^{\text {nd }} \\
\text { surf. }\end{array}$ & $\begin{array}{c}11^{\text {st }} \\
\text { surf. }\end{array}$ & $\begin{array}{c}2^{\text {nd }} \\
\text { surf. }\end{array}$ \\
\hline \multicolumn{9}{|c|}{ flat transparent layer } \\
\hline $0 \%$ & 0.00 & 0.05 & 0.00 & 0.05 & 0.00 & 0.05 & 0.01 & 0.05 \\
\hline $\begin{array}{l}10 \\
\%\end{array}$ & 3.07 & 7.27 & 4.21 & 7.09 & 3.08 & 7.11 & 2.44 & 6.65 \\
\hline $\begin{array}{c}20 \\
\%\end{array}$ & 5.35 & 14.52 & 7.55 & 14.40 & 5.19 & 14.58 & 4.29 & $\begin{array}{c}13.3 \\
1\end{array}$ \\
\hline time & \multicolumn{2}{|c|}{$2.6 \mathrm{~s}$} & \multicolumn{2}{|c|}{$1.6 \mathrm{~s}$} & \multicolumn{2}{|c|}{$3.2 \mathrm{~s}$} & \multicolumn{2}{|c|}{$27.3 \mathrm{~s}$} \\
\hline \multicolumn{9}{|c|}{ wavy transparent layer } \\
\hline $0 \%$ & 1.12 & 2.17 & 1.18 & 3.95 & 1.15 & 2.31 & 1.14 & 1.39 \\
\hline $\begin{array}{l}10 \\
\%\end{array}$ & 5.58 & 10.20 & 7.56 & 11.42 & 5.49 & 11.31 & 4.57 & 8.15 \\
\hline $\begin{array}{l}20 \\
\%\end{array}$ & 10.54 & 19.48 & 15.23 & 21.90 & 10.25 & 18.73 & 8.39 & $\begin{array}{c}15.7 \\
1\end{array}$ \\
\hline time & \multicolumn{2}{|c|}{$2.6 \mathrm{~s}$} & \multicolumn{2}{|c|}{$1.6 \mathrm{~s}$} & \multicolumn{2}{|c|}{$3.2 \mathrm{~s}$} & \multicolumn{2}{|c|}{$27.3 \mathrm{~s}$} \\
\hline
\end{tabular}


which improves the precision of surface measurement, as shown by the

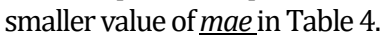

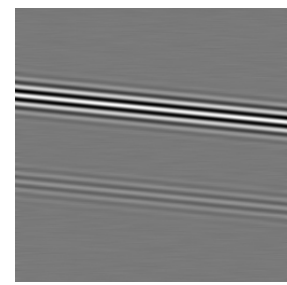

(a)

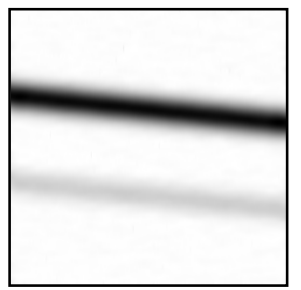

(c)

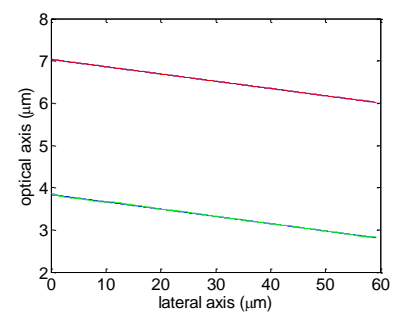

(b)

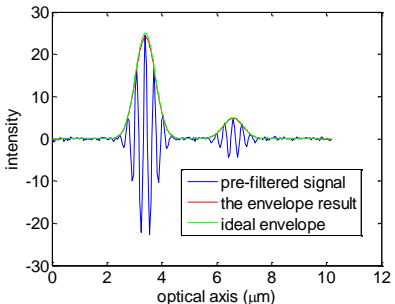

(d)
Fig. 15. Analysis of a synthetic flat transparent layer of constant thickness on a flat substrate: (a) output of pre-filtering using EMDSGolay filter; (b) surface profile, (c) 2D and (d) 1D fringe envelope obtained by TKEO.

Regarding the main step of our procedure i.e. the fringe envelope detection, we observe that TKEO (called " $\mathrm{K}$ ") is a competitive algorithm in terms of robustness and computational time. TKEO provides better surface extraction than the Hilbert Transform (algorithm called "I") and FSA (algorithm called "J"). In the case of the flat transparent layer, TKEO provides the most precise results for the $1^{\text {st }}$ surface extraction and close to the performance of CWT (called "L") for the buried interface ( $2^{\text {nd }}$ surface) extraction, corresponds to the smallest mean absolute error value as shown in Table 4. For the wavy transparent layer, the most precise measurement is provided by CWT, while the performance of TKEO is closer to CWT than the others. However, TKEO has the advantage of being more compact computationally so TKEO is far better in terms of computational time than CWT. For instance, TKEO taking 6.5 $\mathrm{s}$ while CWT taking $30.6 \mathrm{~s}$ in the context of a noisy fringe signal with $\sigma=$ $20 \%$.

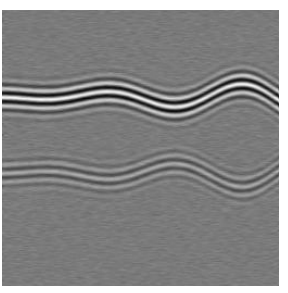

(a)



(C)

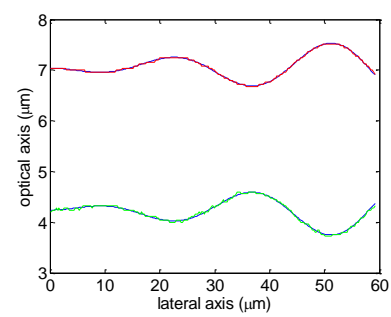

(b)

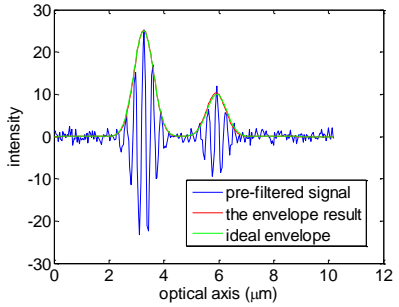

(d)
Fig. 16. Signal processing analysis for synthetic transparent curved surface: (a) output of pre-filtering using EMD-SGolay filter; (b) surface profile, (c) 2D and (d) 1D fringe envelope obtained by TKEO.

\section{B. Performance comparison for the real image}

Finally, we studied the performed of the algorithms using real data, i.e. the fringe image of the resin layer on $\mathrm{Si}$ ( $570 \times 111$ pixels), as shown in Fig. 17(a). This real data was taken on a Leitz-Linnik interference microscope with the following optical parameters: objective $\mathrm{x} 50$, numerical aperture 0.85 , pixel size of $0.113 \mu \mathrm{m}$, and effective average wavelength of $720 \mathrm{~nm}$. The step height of the piezo scanner for scanning the sample along the optical axis is $90 \mathrm{~nm}$ over a dynamic range of 10 $\mu \mathrm{m}$. The image in Fig. 17(a) clearly shows the presence of large variations in the offset along the optical axis near to the edges of the resin layer due to optical effects. In order to eliminate this background on the fringe image, pre-filtering is performed using the EMD technique combined with the Savitzky-Golay filter. The results in Fig. 17(b) show how this pre-filtering approach effectively eliminates the variation in background intensity, while the intensity values of the fringe signals have not been degraded.

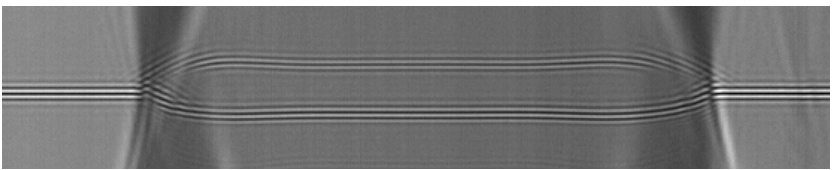

(a)

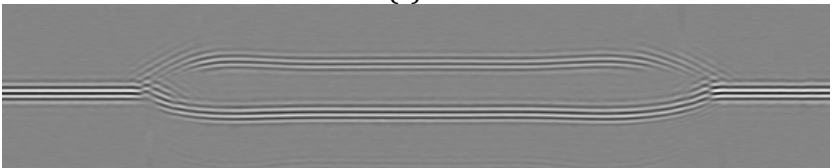

(b)

Fig. 17. (a) real image and (b) output of pre-filtering using EMD-SGolay filter.

Then, the different envelope detection techniques were performed to obtain the fringe envelope of the pre-filtered fringe signals. Figures 18(a-d) and Figures 19(a-d) compare the resulting fringe envelopes and surface profiles extracted by HT, FSA, CWT, and TKEO.

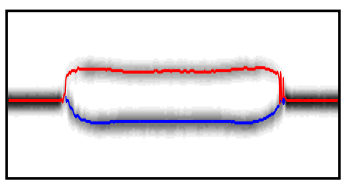

(a)

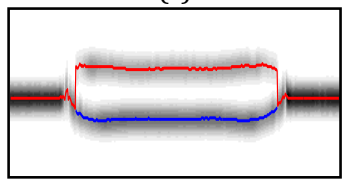

(c)



(b)

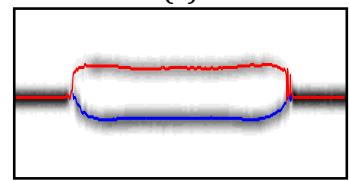

(d)

Fig. 18. 2D envelope peak detection obtained by: (a) HT, (b) FSA, (c) CWT, (d) TKEO.

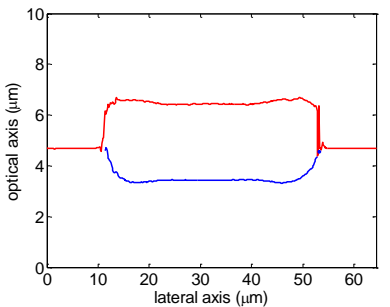

(a)

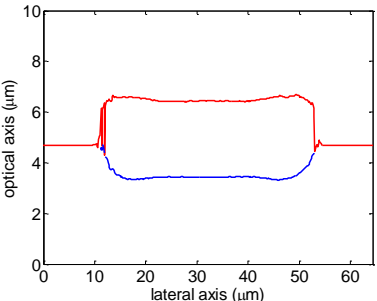

(b) 


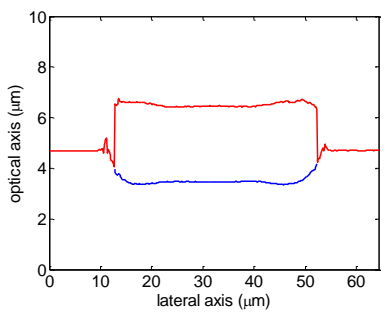

(c)

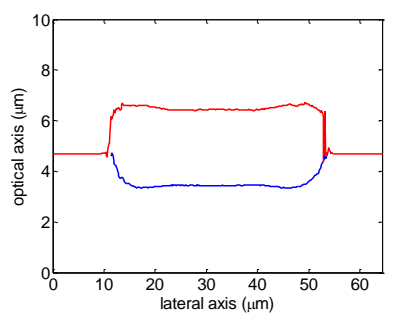

(d)
Fig. 19. Surface profile obtained using: (a) HT, (b) FSA, (c) CWT, (d) TKEO.

A performance comparison of the different envelope detection techniques is represented by the value of the roughness parameter, i.e. the maximum height of the profile $R_{t}$, and the roughness average $R_{a}$, as shown in Table 5 . We have evaluated four different regions of interest (ROI) of the surface profile: ROI- $1=0-9 \mu \mathrm{m}$; ROI- $2=20-45 \mu \mathrm{m}$ on the $1^{\text {st }}$ surface; ROI-3 $=55-64 \mu \mathrm{m} ; \mathrm{ROI}-4=20-45 \mu \mathrm{m}$ on the $2^{\text {nd }}$ surface, (Fig. 20). The smaller the value of this roughness parameter is the better is the performance of the algorithm, in terms of sensitivity.

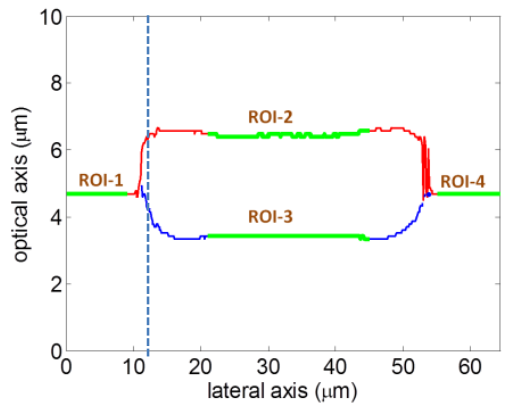

Fig. 20. Region of interest (ROI)

Based on the table, for the single surfaces (ROI-1 and ROI-3), all the algorithms show excellent performance, as illustrated by the very small value of $R_{t}=18.0-27.0 \mathrm{~nm}$ and $R_{a}=2.8-6.2 \mathrm{~nm}$. Then for the transparent surface regions (ROI-2 and ROI-4), the algorithms seem to behave similarly according to the value of the roughness parameters ( $R t$ and $R a)$, which do not differ significantly. The different envelope techniques provide similar performance for the single surface as well as for the transparent surface, since the fringe image is slightly noisy. In accordance with the results of performance comparison based on synthetic images, the performances of the different envelope detection techniques depend on the SNR in the fringe image, while their performances are similar for the noiseless fringe image.

Table 5. Experiment result using pre-filter 3 and different envelope detection techniques (nm)

\begin{tabular}{|c|c|c|c|c|}
\hline & ROI-1 & ROI-2 & ROI-3 & ROI-4 \\
\hline axis/length & $(0-9 \mu \mathrm{m})$ & $\begin{array}{c}(20-45 \\
\mu \mathrm{m})\end{array}$ & $\begin{array}{c}(55-64 \\
\mu \mathrm{m})\end{array}$ & $\begin{array}{c}(20-45 \\
\mu \mathrm{m})\end{array}$ \\
\hline & \multicolumn{4}{|c|}{$\mathrm{HT}$} \\
\hline$R t(\mathrm{~nm})$ & 18.0 & 99.0 & 27.0 & 63.0 \\
\hline \multirow[t]{2}{*}{$R a(\mathrm{~nm})$} & 4.8 & 16.5 & 5.7 & 8.8 \\
\hline & \multicolumn{4}{|c|}{ FSA } \\
\hline$R t(\mathrm{~nm})$ & 18.0 & 108.0 & 27.0 & 63.0 \\
\hline \multirow[t]{2}{*}{$R a(\mathrm{~nm})$} & 4.6 & 15.8 & 5.3 & 8.6 \\
\hline & \multicolumn{4}{|c|}{ CWT } \\
\hline$R t(\mathrm{~nm})$ & 18.0 & 108.0 & 27.0 & 63.0 \\
\hline \multirow[t]{2}{*}{$R a(\mathrm{~nm})$} & 2.9 & 16.7 & 6.2 & 8.2 \\
\hline & \multicolumn{4}{|c|}{ TKEO } \\
\hline$R t(\mathrm{~nm})$ & 18.0 & 108.0 & 27.0 & 63.0 \\
\hline$R a(\mathrm{~nm})$ & 2.8 & 17.3 & 5.9 & 8.3 \\
\hline
\end{tabular}

\section{CONCLUSION}

In this paper, a comparative performance study, based on common signal processing techniques, has been performed to analyse white light fringes in CSI, namely the HT, CWT, FSA and TKEO algorithms. We have evaluated three different types of pre-filtering techniques which combine the EMD and classic cantered derivative and the Wiener/Savitzky-Golay filter in order to process simultaneously the noise and the offset component. A quantitative comparison of our results based on synthetic and real fringe images highlights the efficiency of such pre-filtering/filtering combination regarding other pre-filtering methods. In addition, pre-filtering with EMD maintains the original amplitude of the signal, making it more precise in identifying the fringe envelope peak in the case of low contrast fringes. Moreover, we have shown that the use of cubic spline smoothing and Gaussian curve 
fitting is helpful to remove the artifacts and determine more precisely the fringe envelope peak position.

To evaluate the performance of the different fringe envelope detection techniques, 12 algorithms (A-L) using different pre-filtering and envelope detection techniques have been tested. Based on the simulation results using two synthetic XZ fringe images (flat and undulating transparent layers on silicon substrates), it has been shown that TKEO $(\mathrm{K})$ and CWT are more competitive in terms of surface extraction than the HT and FSA algorithms. Therefore TKEO is slightly better in terms of computational time.

Acknowledgment. The authors would like to acknowledge the Indonesian Ministry of Trade for financial support of the doctoral work of G. Gianto.

\section{References}

1. P. C. Montgomery, F. Salzenstein, D. Montaner, B. Serio, and P. Pfeiffer, Proc. SPIE 8788 (2013). pp. 87883G-87883G-11.

2. J. Schmit, J. Reed, E. Novak, and J. K. Gimzewski, Journal of Optics A: Pure and Applied Optics 10 (2008).

3. M. Conroy and J. Armstrong, Journal of Physics Conference Series $\mathbf{1 3}$ (2005).

4. J.M. Schmitt, IEEE Journal of Selected Topics in Quantum Electronics 5, No. 4 (1999).

5. A Dubois, G Moneron, K Grieve and A C Boccara, Physics in Medicine and Biology 49, 7 (2004).

6. F. Salzenstein, P. Montgomery, and A. O. Boudraa, Optical Society of America 22 (2014). pp. 18325-18334.

7. E. Halter., P.C. Montgomery, D. Montaner, R. Barillon, M. del Nero, C. Galindo, and S. Georg, Applied Surface Science 256, 6144 (2010).

8. P.J. Caber, Applied Optics 32 (19), 3438-3441 (1993).

9. K.G. Larkin, J. Opt. Soc. Am. A 18, No. 8 (2001)

10. G.S. Kino and S.S.C. Chim, Applied Optics 29: 3775 (1990).

11. K. Larkin, JOSA A 13, 832-843 (1996).

12. P. Sandoz, Opt. Lett. 22, 1065-1067 (1997).

13. T. Suzuki, H. Matsui, S. Choi, and O. Sasaki, Optics Communications 13 (2013). pp 172-176.

14. A. Dubois, L. Vabre, A.C. Boccara, and E. Beaurepair, Applied Optics 41 No.4 (2002).

15. A. Boudraa, F. Salzenstein, and J. Cexus, Opt.Eng. 44, 7001-7010 (2005).

16. P. Flandrin, P. Goncalves, and G. Rilling, Proc. of $12^{\text {th }}$ European Signal Proc. Conf., (2004). pp. 1581-1584.

17. Z.K. Peng, F.L. Chu, Mech. Syst. Sig. Process. 18(2). 199-221 (2004).

18. H. Adeli, Z. Zhou, N. Dadmehr, Journal of Neuroscience Methods 123:69-87 (2003).

19. P. D. Swami, R. Sharma, A. Jain, D.K. Swami, Speech Communication 70, 1-12 (2015).

20. L. Huang, Q. Kemao, B. Pan, A. K. Asundi, Optics and Lasers in Engineering 48:141-148 (2010).

21. J. Sokolovsky, Y.Yitzhaky, and I.Abdulhalim, Applied Optics 51:8390 (2012).

22. K. M. Medicus and M. J. Jansen, Proceedings of the ASPE Annual Meeting (2010).

23. J. F. Kaiser, Proc. IEEE Int. Conf. Acoustics, Speech, and Sig. Process. 3 (1993). pp.149-152.

24. P. Maragos, T. Quatieri, and J. F. Kaiser, Proc. IEEE Int. Conf. Acoustics, Speech, and Sig. Process. (1991). pp.421-424.

25. P. Maragos, T. F. Quatieri, and J. F. Kaiser, IEEE Trans. Signal Process. 41:3024-3051 (1993).

26. F. Salzenstein, P.C. Montgomery, D. Montaner, and A. Boudraa, J. Appl. Sig. Proc. 17:2804-2815 (2005).

27. J. C. Cexus and A. O. Boudraa, Int. Journal of Sig. Process. 1, (2004). pp.1-5.

28. Standards: ASME B46.1-1995, ASME B46.1-1985, ISO 4287-1997, ISO 4287/1-1997.

29. Standards: ASME B46.1-1995, ISO 4287-1997.
30. A.O. Boudraa, J.C. Cexus, and Z. Saidi, International Journal of Signal Processing 1 (2004). pp. 33-37.

31. J. S. Lim, Two-Dimensional Signal and Image Processing, Prentice Hall (1990). P.527.

32. S. J. Orfanidis, Introduction to Signal Processing, Prentice Hall (1996). P.427.

33. F. Yang and X. He, Appl. Opt. 46, 7172-7178 (2007). 